\title{
IMMUNOTHERAPY
}

\section{T cells pass on the baton during checkpoint inhibition}

Immune-infiltrated tumours respond better to immune checkpoint inhibition than immune-desert tumours, which could be due to therapy-induced expansion of pre-existing tumour-specific T cell clones. Now, Yost, Satpathy et al. show that immune checkpoint blockade can lead to expansion of $\mathrm{T}$ cell clones that are distinct from pre-existing T cell clones, a phenomenon termed clonal replacement, in patients with basal cell carcinoma (BCC) or squamous cell carcinoma (SCC).

The researchers performed singlecell RNA sequencing (scRNA-seq) and $\mathrm{T}$ cell receptor sequencing (scTCR-seq) on tumour biopsysamples from 11 patients with advanced BCC before and after treatment with antiprogrammed cell death 1 (PD1) antibodies. The tumours showed an immunological response to anti-PD1 treatment consisting of increased $\mathrm{T}$ cell infiltration and mutational loss. Cluster analysis of scRNA-seq and scTCR-seq data identified 19 cell clusters of immune and stromal cells. Infiltrating immune cells clustered together regardless of which patient they were derived from. Malignant cells showed patient-specific copy number variations and clustered together based on the BCC subtype and the patient they were derived from. In addition, gene expression profiles of tumours from different

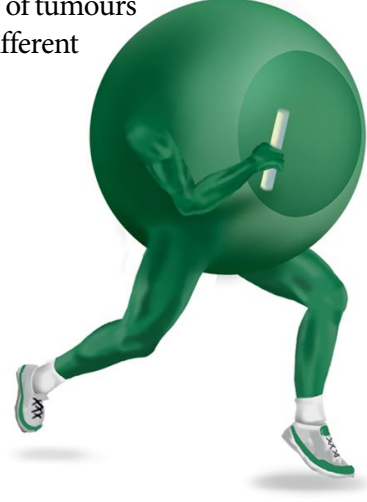

patients showed that up to 577 genes were differentially expressed. Thus, immune cell phenotypes were consistent between different tumour microenvironments, even though there were substantial levels of intertumoural heterogeneity.

Focusing on the T cell response to anti-PD1 treatment, the researchers identified nine distinct $\mathrm{T}$ cell clusters, consisting of $\mathrm{T}$ cells from different patients before or after treatment. Within these clusters, there was an increased frequency of activated, chronically activated (referred to as exhausted) and intermediate activated or intermediate exhausted $\mathrm{CD} 8^{+}$ T cells after treatment with antiPD1. Using diffusion maps, the researchers identified two components in the relationship between $\mathrm{CD}^{+} \mathrm{T}$ cell clusters, with the first component (correlated with the T cell exhaustion-associated genes PDCD1 and HAVCR2) separating activated from exhausted $\mathrm{T}$ cells and the second component (correlated with the $\mathrm{T}$ cell activationassociated genes IFNG and TNF) separating activated and exhausted $\mathrm{T}$ cells from naive and memory $\mathrm{T}$ cells. Grouping of cells by treatment status revealed an increased frequency of activated and exhausted T cells in tumours after treatment.

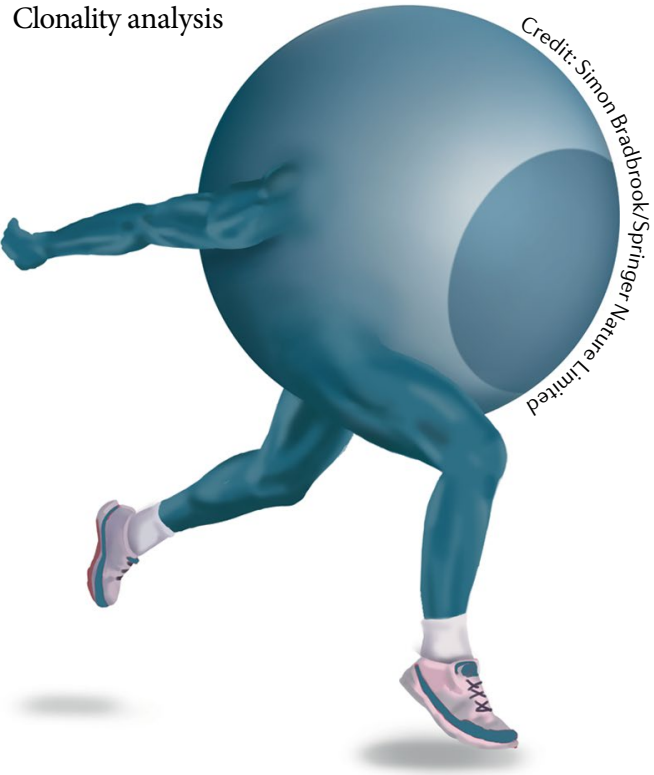

using scTCR-seq data revealed that exhausted $\mathrm{CD}^{+} \mathrm{T}$ cells had the highest levels of clonality compared with all other $\mathrm{CD} 8^{+} \mathrm{T}$ cells. Using activation and exhaustion signatures generated based on the top 50 genes correlated with IFNG or HAVCR2 expression, the researchers found that high expression of the exhaustion signature was associated with tumour reactivity and high proliferation, and was present in the largest $\mathrm{CD}^{+} \mathrm{T}$ cell clone population. When cells were grouped by clonotype or TCR specificity, they were more likely to share a common phenotype than when they were grouped randomly, indicating that clonally expanded $\mathrm{T}$ cells share a common phenotype that correlates with antigen specificity and is stable in response to anti-PD1.

A small population of exhausted $\mathrm{CD}^{+} \mathrm{T}$ cells expressed the transcription factor TCF7, marking stemlike $\mathrm{T}$ cells that are known to expand in response to anti-PD1. However, only a minority of post-treatment exhausted clones in this analysis was derived from TCF7 ${ }^{+}$ clones. By contrast, $84 \%$ of exhausted $\mathrm{T}$ cell clones detected after anti-PD1 treatment were derived from clones that had not been detected before treatment. Indeed, across all patients, the majority of these exhausted clones were derived from expansion of novel clones (clonal replacement). Of note, one-third of the novel exhausted clonotypes were detected in peripheral blood, indicating a possible approach to monitor tumour-specific $\mathrm{T}$ cell responses to anti-PD1 treatment. Analysis of scRNA-seq and scTCR-seq data from tumour biopsy samples of patients with SCC before and after treatment with anti-PD1 also showed the described features of clonal replacement.

Thus, this study suggests that the response to anti-PD1 therapy relies on the intrinsic ability of tumours to recruit novel $\mathrm{T}$ cell clones, which replace pre-existing exhausted $\mathrm{T}$ cells that have limited capacity to reinvigorate in response to anti-PD1.

Ulrike Harjes

ORIGINAL ARTICLE Yost, K. E. et al. Clonal replacement of tumor-specific T cells following PD-1 blockade. Nat. Med. 25, 1251-1259 (2019) 